\title{
Novel Sampling Algorithm for Levy-Walk Based Mobile Phone Sensing
}

\author{
Thejaswini $\mathrm{M}^{\dagger}$, P. Rajalakshmi ${ }^{\dagger \dagger}$, U. B. Desai ${ }^{\dagger \dagger}$ \\ ${ }^{\dagger}$ Department of Computer Science and Engineering \\ ${ }^{\dagger}$ Department of Electrical Engineering \\ Indian Institute of Technology Hyderabad, India \\ Email: (cs10p003, raji, ubdesai)@iith.ac.in
}

\begin{abstract}
Mobile phones or smart phones equipped with different communication technologies and sensors have become pervasive application development platform for opportunistic and human-centric sensing. Optimisation of battery energy consumption and opportunistic sensing coverage are important issues under mobile phone sensing. This paper proposes a simple sampling algorithm based on human-walk velocity for mobile phone sensing. We analyse the impact of human-walk velocity on battery energy consumption and spatial coverage for mobile phone sensing by considering general regular sampling of sensors and proposed sampling method. When Levy walk mobility parameter $\alpha=1$, the proposed sampling algorithm shows better performance in terms of both spatial coverage and reduction of battery energy consumption for mobile phone sensing activity.
\end{abstract}

\section{INTRODUCTION}

Latest mobile phones or smart phones are becoming ubiquitous application development platform for the Internet of Things (IoT), participatory sensing and crowd-sourced sensing, with different types of sensors like camera, microphone, GPS, temperature, accelerometer and communication technologies like Wi-Fi, Bluetooth, Near Field Communication (NFC) embedded with them [1], [2], [3]. Sensors embedded mobile phones can be used for monitoring different environmental factors like temperature, humidity, urban noise pollution monitoring, carbon footprint, air pollution monitoring and urban traffic monitoring.

Human mobility enables collection of aggregated and nonaggregated mobile phone sensor data by forming co-operative sensing task with surrounding neighbors, or individual users mobile phone may simply send its sensor data to designated destinations like central or cloud servers to do processing, aggregation, and mapping of sensor data [1], [3], [4]. In both the cases sensing task assignment to participating users and sending sensor data to designated destination can be done through ad-hoc or infrastructure oriented networking.

In former case, formation of co-operative mobile phone sensing task requires regular lookup for neighbors, processing and exchange of messages, security and trust protocols. Dynamic and unpredictable human mobility nature and activities affects the performance of co-operative mobile phone sensing task. The later type of mobile phone sensing activity can be useful for sparsely populated and dynamically changing human networks, where availability of neighbors is sparse or changing in a short period. Our work is concerned with the later case i.e mobile phone sensing at individual user level, which is also useful when heterogeneous sensing task assignment for each individual users is required.

Whatever may be the scale of mobile phone based sensing applications, for continuous and regular interval of sensing activity, optimisation of energy consumption is an important issue under human-centric and opportunistic sensing. There has been enough theoretical and practical work done on optimization of energy consumption and improving sensing area coverage for both static and mobile networks by using Random waypoint (RWP) mobility model [5] [6]. In this paper, we assume that each individual user mobile phone sensing task is carried out without the knowledge of neighbor sensing tasks for given period of time. We concentrate on analysing sensing area coverage and reduction of battery energy consumption for sensing activity at individual user level. We concentrate only on spatial coverage of mobile phone sensors and not on temporal coverage.

Mobile phone sensing area coverage depends on sensing range and velocity of human-walk. Levy walk (LW) depict statistical properties of human mobility patterns [7], [8], [9], [10], [11]. Authors of [7], [8] describes the human walk mobility model using real traces and report that independent of geographical constraints, the heavy-tail tendency of flight length distribution, super and sub-diffusive mean-square displacement features must be inherent in human walk mobility models. The description of LW mobility model is given section II. Authors of [9] explore dynamic graph properties under LW mobility model. Decrease of LW mobility parameter $\alpha$ (Section-II) leads to dynamic human networks [9]. We use LW mobility model given in [12] for the simulation of proposed sampling algorithm. Authors of [13] discusses reducing number of participatory users plus improving coverage by assuming that users are aware of their path. Authors of [4] presents collaborative mobile phone sensing using cloud network. They assume that mobile phone users path is known in advance. In our work we analyse the impact of dynamic human movements and patterns on spatial coverage for mobile phone sensing at individual user level.

We propose a simple sampling algorithm based on humanwalk velocity and compare how the varying velocity of humanwalk affects the spatial coverage and energy consumption. The results shows that mobile phone sensor sampling with respect 
to users walking velocity will reduce spatial overlap of sensing area and reduce energy consumption of sensing process. The results will be helpful in designing continuous and regular mobile sensing applications.

The remainder of this paper is organized as follows. Section II describes system model for the proposed work. Section III gives description of proposed sampling algorithm for mobile phone sensing. Section IV discusses evaluation of simulation results and section $\mathrm{V}$ concludes the paper.

\section{SYSTEM MODEL}

This section provides the description of models used for the development of proposed work.

\section{A. Levy-Walk Mobility Model}

Human walk patterns closely resembles LW patterns [8]. LW is used for modeling animal foraging patterns [14]. In LW mobility model a step is represented by four variables ( $\left.L, \Theta, \Delta T_{f}, \Delta T_{p}\right)$ [8], [9]. $L$ is length of flight (flight is defined as straight line trip without pause or change of direction), drawn from power law distribution with parameter $\alpha$ and its probability density function (PDF) [8] is given by:

$$
\begin{aligned}
p(L) \propto|L|^{-(1+\alpha)}, & L \in\left(L_{\min }, L_{\max }\right) \\
& 0<\alpha<2
\end{aligned}
$$

$L_{\min }$ and $L_{\max }$ are minimum and maximum flight length. At the beginning of each flight, LW node chooses a direction with angle $\Theta$ randomly from a uniform distribution within range $[0,2 \pi] . \Delta T_{f}[8]$ is flight time, the time taken by LW nodes to complete the flight length and is given by below equation:

$$
\Delta T_{f}=\tau L^{(1-v)}, 0 \leq v \leq 1
$$

$\tau$ and $v$ are constants. $\Delta T_{p}$ is pause time, drawn from power law distributions with parameter $\beta$ and its PDF [8] is given by:

$$
\begin{gathered}
p\left(\Delta T_{p}\right) \propto\left|\Delta T_{p}\right|^{-(1+\beta)}, \quad \Delta T_{p} \in\left(T_{\text {pmin }}, T_{\text {pmax }}\right) \\
0<\beta<2
\end{gathered}
$$

$T_{p \min }$ and $T_{\text {pmax }}$ are minimum and maximum pause time. The levy distribution [8] is given in equation (4).

$$
f_{Y}(y)=\frac{1}{2 \pi} \int_{-\infty}^{+\infty} e^{-i t y-|a t|^{b}} d t
$$

where $a$ and $b$ are scale factor and exponent parameter respectively.

Mobile phones are also termed as mobile nodes. We assume that mobile phones use embedded GPS for getting location co-ordinates and also embedded with required sensors. Let $A$ be the communication area and $M$ be a set of mobile nodes. Simulation duration is represented by $T$.

Consider single mobile phone user $M_{1}$, where $M_{1} \in M$. Let $p$ be the total number of sensors embedded to a mobile phone $M_{1}$ and $r_{s(i)}$ be the sensing range of $s(i)^{t h}$ sensor, where $i=1,2, \ldots, p$. We consider disk model for sensing range of each sensor. Let $t_{s(i)}$ be the sampling interval of $s(i)^{t h}$ sensor.
When mobile node moves in a straight line path with constant velocity $v$ and without pause or change of direction, then $t_{s(i)}$ for non-overlap sensing coverage with respect to $s(i)^{t h}$ sensor is given by:

$$
t_{s(i)}=2 * \frac{r_{s(i)}}{v}
$$

Let average or preferred human walk velocity be $v_{h w}$. Under LW mobility model equation (5) is given by:

$$
t_{s(i)}=2 * \frac{r_{s(i)}}{v_{h w}}
$$

We consider energy consumed by mobile phone battery for sensing and processing a sensor sample $\delta$ to be one unit and energy consumed for a location sample $\ell$ also to be one unit. $\lambda_{s(i)}$ gives the total number of samples over simulation duration $T$ with respect to $s(i)^{t h}$ sensor. The total energy consumed for sensor and location samples over $T$ for single mobile phone $s(i)^{t h}$ sensor is given by equation (8) and equation (9) respectively. Equation (10) gives sum of total energy consumed by all the sensors embedded to a mobile phone including GPS.

$$
\begin{gathered}
\lambda_{s(i)}=\frac{T}{t_{s(i)}} \\
\phi_{s(i)}=\sum_{n=1}^{\lambda_{s(i)}} \delta_{n} \\
\hbar_{s(i)}=\sum_{n=1}^{\lambda_{s(i)}} \ell_{n} \\
E=\sum_{s(i)=1}^{p} \phi_{s(i)}+\sum_{s(i)=1}^{p} \hbar_{s(i)}
\end{gathered}
$$

\section{Sampling Algorithm For Mobile Phone SENSING}

Human-walk characteristic consists flight truncations and pause, because of human intensions and activities, contexts, home coming, geographical-constrains like buildings, small shops, trees, street structure and boundaries [7], [8]. Whatever may be the cause of pause and flight truncations, in real-time they are unpredictable. If we follow equation (6) for mobile phone based sensor sampling, flight truncations and pause time causes spatial overlaps. As opposed to Random Way Point (RWP) mobility model (occurrences of long flights is more), LW mobile nodes hardly move in straight line path and consists of flight truncations [7], [8]. To reduce spatial overlap under LW mobility model we propose a simple sampling algorithm based on human-walk velocity. We assume that mobile phones have enough memory space to store sensed location points. The pseudocode of sampling algorithm for mobile phone sensing is given Algorithm 1.

We can assign $v_{h w}$ value according to the person walking context (walking slow, walking fast, jogging or running). Also it is very easy to program to get adaptive $v_{h w}$ value, according to changing person walking context. Preferred human 


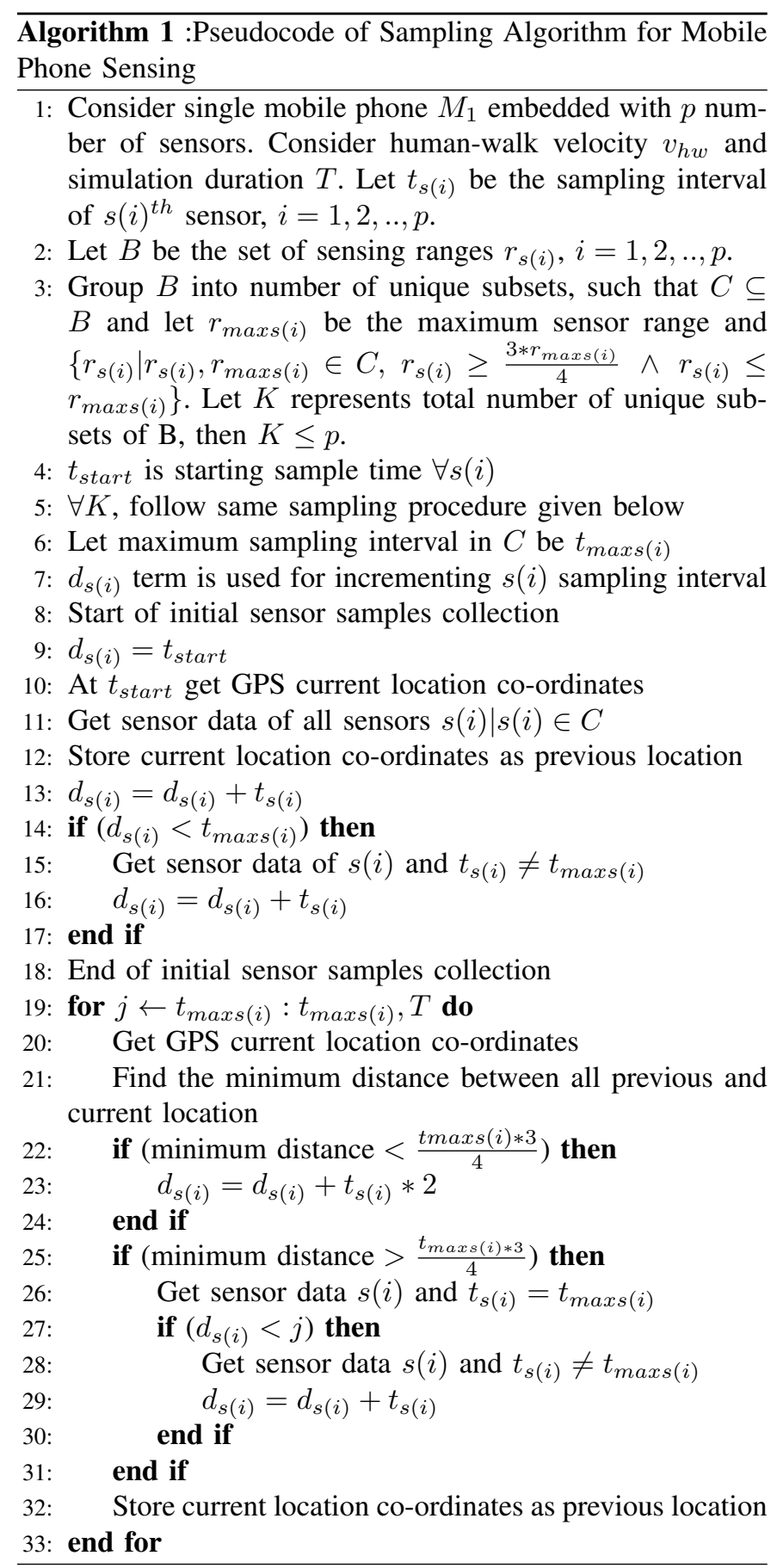

walking speed is $\approx 1$ meter/second [15]. For simplifying the performance analysis, in our simulations we set $v_{h w}=$ 1 meter/second. In Algorithm 1, a mobile phone sensor's range $r_{s(i)}$ are grouped into unique subsets $K(K \leq p)$, such that in each subset, sensor's range differ with respect to each other by $\leq \frac{3}{4}$. Example, if a mobile node consists of 5 sensors and their sensing ranges are represented in order, say $B=\{10,13,15,17,20\}$, then we can group $B$ into unique subsets, $\{10,13\}$ and $\{15,17,20\}$. We reduce the number of sensed location samples by grouping sensors into $K$ number

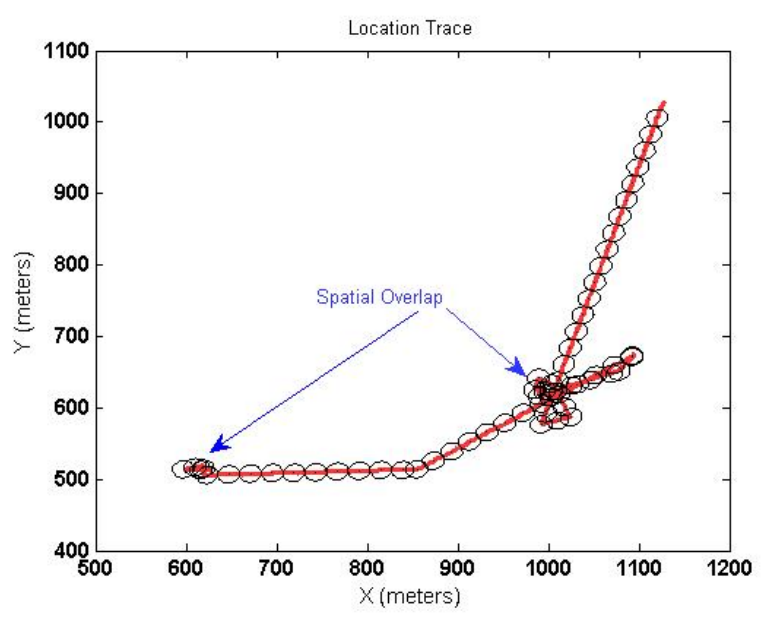

Fig. 1. General Sampling Method For Mobile Phone Sensing

of unique subsets and in each subset, only for the maximum sensor range $r_{\max (i)}$, location samples are considered with corresponding sensor samples. Mobile nodes store sensed location points. By calculating the disatnce between previous and currect locations, we reduce the spatial overlap caused by LW mobile nodes pause or flight truncations. If minimum distance calculated between all previous sensed location samples and current location sample is less than $\frac{3 * t_{\operatorname{maxs}(i)}}{4}$ (as we assumed $v_{h w}=1$ meter/second), then current sensing process is skipped for all the sensors of that particular subset. If total number of unique subsets $K<p$, equation (9) and (10) is changed as shown in equation (11) and (12) respectively.

Figure 1 and 2 show location trace of single mobile node for duration of 1500 seconds with maximum and minimum pause time set to 10-20 seconds. Assumed range of sensor is $r_{s}=12$ meters. Other considered parameters are shown in Table 1. In Figure 1 and 2, circles represent sensing range. Overlaps of circles in Figure 1 represents spatial overlap of sensing area due to either flight truncation or pause. Figure 2 shows reduction in number of spatial overlap of sensing area for the same trace after applying proposed sampling algorithm.

$$
\begin{aligned}
& \hbar_{s(i)}=\sum_{n=1}^{\lambda_{s(i)}} \ell_{n}, i=1,2, \ldots K \\
& E=\sum_{s(i)=1}^{p} \phi_{s(i)}+\sum_{s(i)=1}^{K} \hbar_{s(i)}
\end{aligned}
$$

\section{Simulation Results AND Discussion}

In this section, we analyse proposed sampling algorithm for mobile phone sensing under LW mobility model in terms of average battery energy consumed and spatial coverage. We also analyse the effect of LW mobility $\alpha$ parameter on mobile node average velocity and area coverage in terms of coverage ratio. We consider 3 different, low, high and medium values for $\alpha$, i.e $0.1,1.9$ and $1(0<\alpha<2)$ respectively. To calculate 


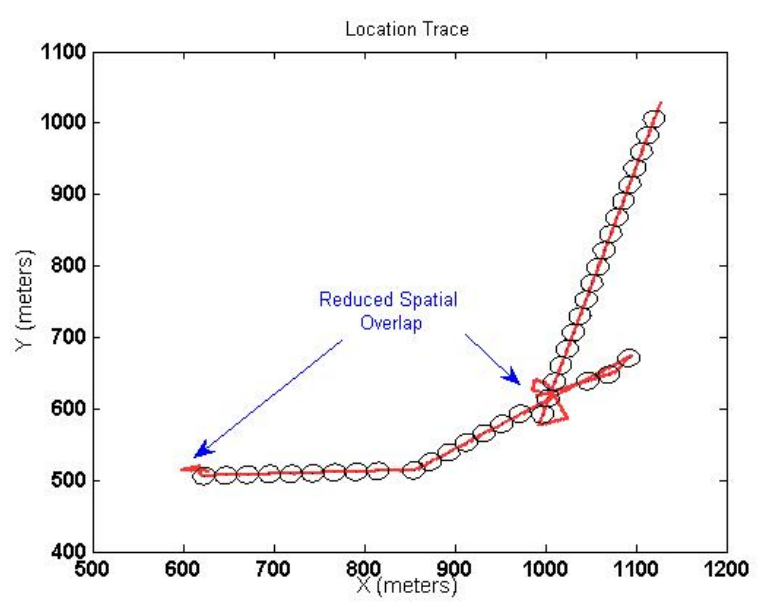

Fig. 2. Proposed Sampling Method For Mobile Phone Sensing

TABLE I

SIMULATION PARAMETERS

\begin{tabular}{|c|c|}
\hline Simulation Area $A$ & $1.2 \mathrm{Km} * 1.2 \mathrm{Km}$ \\
Flight parameter $\alpha$ & $1,1.9,0.1$ \\
Pause parameter $\beta$ & 1.0 \\
Average flight length & $5-1200$ meters \\
Pause time & $1-2$ seconds \\
$v_{h w}$ & 1 meter/second \\
Location sample interval $t_{l s}$ & 1 second \\
Range of sensor1, sensor2, sensor3 & $20,17,15$ meters \\
\hline
\end{tabular}

average velocity of a mobile node we consider average of 500 samples of locations trace and $T$ is set to 16000 seconds. Other considered parameters are shown in Table 1. For $\alpha=$ $0.1,1$ and 1.9 , we got average velocity of a mobile node 2.33 , 2.26 and 2.04 meter/second respectively, and the results reveal that lower the $\alpha$ value, higher will be the average velocity of mobile node.

For calculating the coverage ratio, simulation area $A$ is divided into square grids, where length of square is equal to sensing range $\left(r_{s(i)}\right)$. Other considered parameter values are shown in Table 1 . Coverage ratio of any $s(i)^{t h}$ sensor is defined as the ratio of total number of grids covered at least once by a mobile node at every sample interval $t_{s(i)}$ to the total number of grids [16]. Large value of coverage ratio depicts large area covered by a mobile node within total area.

Figure 3, 4 and 5 show coverage ratio, average units of energy consumed and area coverage for individual user mobile phone sensing with the assumption that mobile phones having a single sensor. For all mobile phones, sensor range is assumed to be 12 meters. Other considered simulation parameters are given in Table 1. We use term GS to represent general sampling method, i.e sampling with regular interval of time (equation (6)). In order to represent proposed sampling method we use term HWS (Human-Walk Sampling). Figure 3 show sensing coverage ratio for different values of $\alpha$. We consider a mobile node walk duration, $T=16000$ seconds. For GS method, sensor data is collected at every $t_{s(i)}$ time, we get

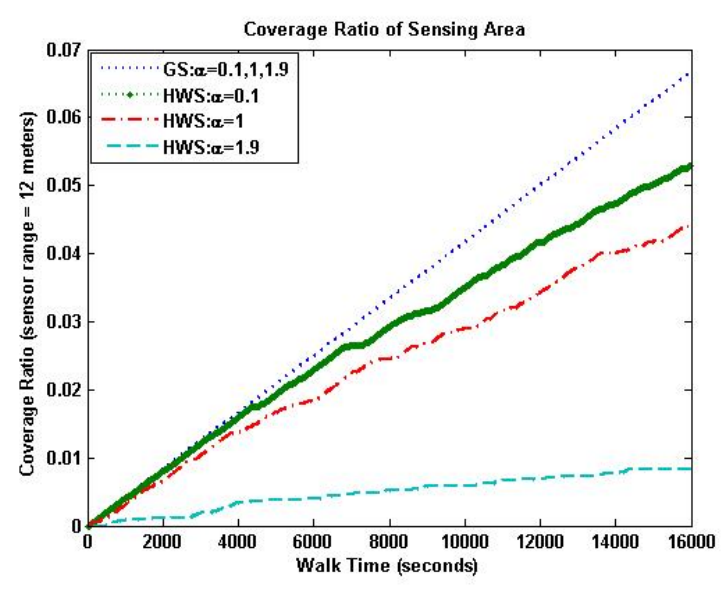

Fig. 3. Coverage Ratio of Sensing Area

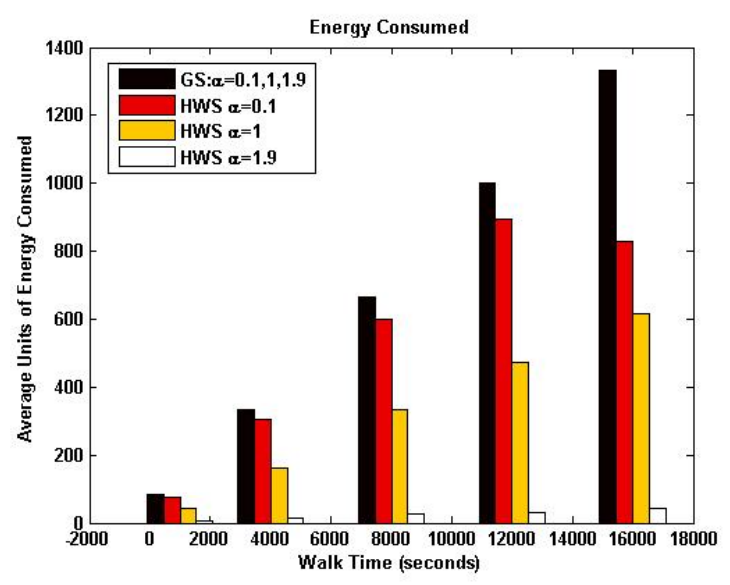

Fig. 4. Average Units of Energy Consumed for Sensing Process

same curve plot for $\alpha=1.0,1$ and 1.9. Figure 3 reveals that due to reduced number of sampling process for HWS method, coverage ratio is less then GS method, and it also depicts that for HWS method, decrease in $\alpha$ value, faster will be increase in coverage ratio. The objective to analyse the performance of GS and HWS sampling methods in terms of coverage ratio is to know only the effect of velocity of mobile nodes on spatial coverage with time. We need better performance in terms of reduction of energy consumption and spatial coverage. For accurate spatial coverage analysis for GS and HWS sampling methods, we use average sensing area coverage in square meters by considering the sensed location co-ordinates and sensor range.

In Figure 4 and 5, for each different values of $\alpha$, we consider average of 500 samples for plotting the results. First we explain GS method performance for varying $\alpha$ values. As in GS, sensor data is collected at every $t_{s(i)}$ time, we get same results for average units of energy consumed for sensing process irrespective of any $\alpha$ value for given walk duration (Figure 4, equation (10)). Coming to HWS performance discussion, 


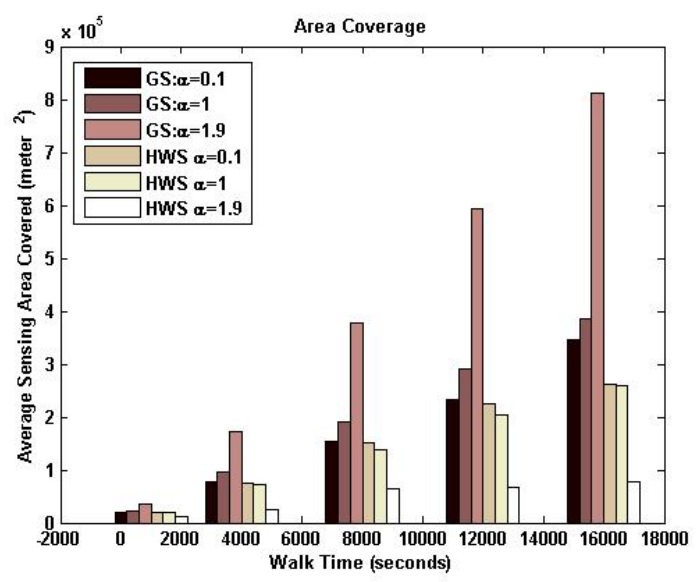

Fig. 5. Average Sensing Area Covered

the proposed method reduces spatial overlaps by reducing number of sampling process. Figure 4 shows that, for proposed HWS method, average energy consumption is reduced for all considered values of $\alpha$, compared to GS method.

For $\alpha=0.1$ to 1 , mobile nodes velocity varies from normal to high. For GS technique sensor coverage area decreases for $\alpha=0.1$ and 1, compared to $\alpha=1.9$ (Figure 5). We require better performance in terms of both spatial coverage and reduction of energy consumption. Combining the results of Figure 4 and 5, when $v_{h w}$ is set to 1 meter/second, GS technique suits for slow walkers. When $\alpha=1$ proposed algorithm HWS shows better performance in terms of both battery energy reduction and coverage of sensing area, so HWS suits well for normal walking speed.

Figure 6, 7 and 8 show performance of HWS and GS sampling methods in terms of coverage ratio, Figure 9 shows performance in terms of average units of energy consumed and Figure 10, 11 and 12 shows performance analysis in terms of average sensing area covered for mobile phone sensing activity with the assumption that each mobile node has three sensors. Considered three sensors are termed by sensor 1 , sensor 2 and sensor3, their sensing ranges and other considered parameters are given in Table 1. In Figure 6, 7 and 8, for HWS sampling method, coverage ratio is faster when $\alpha=0.1$, as velocity of mobile node is high compared to other considered $\alpha$ values.

For Figure 9 to 12, average of 500 simulation runs, each of duration $T=16000$ seconds is considered to plot results. Figure 9 shows average units of energy consumed for mobile phone sensing activity versus walk durations. In Figure 9, for GS technique, there is drastic increase in units of energy consumption for sensing process, as we consider that for each sensor sampling process, mobile phone GPS is used to get current location record. In HWS method we consider that GPS is used only for the maximum range sensor sampling in each subset of sensors. The results of mobile phone multi-sensor sampling activity depict the same conclusion of single sensor sampling activity that, in terms of both energy consumption (Figure 9) and sensing area coverage (Figure 10, 11 and 12),

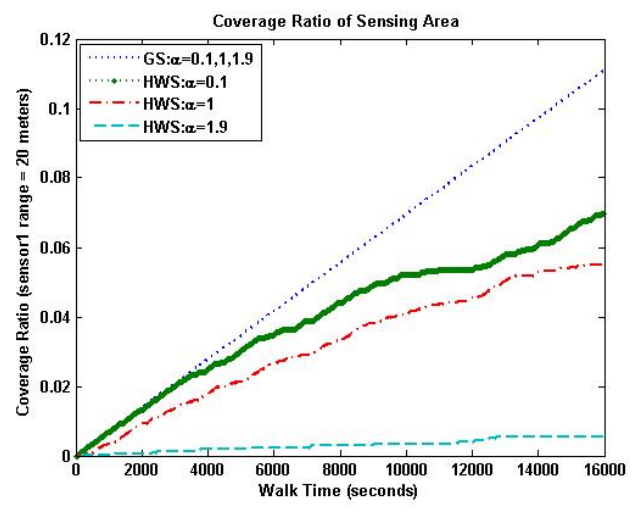

Fig. 6. Coverage Ratio-sensor1

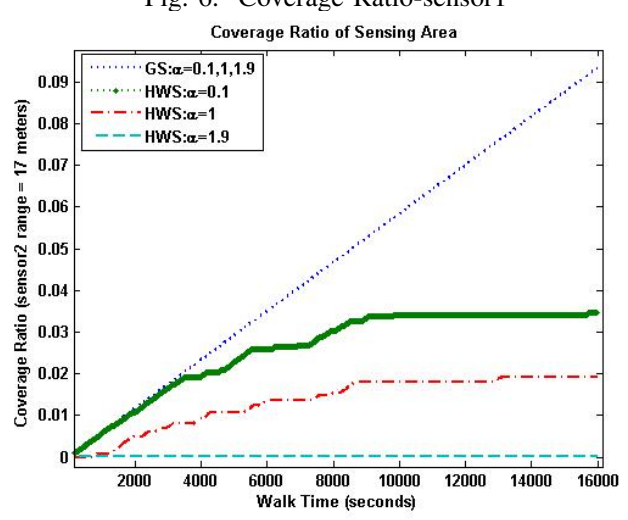

Fig. 7. Coverage Ratio-sensor2

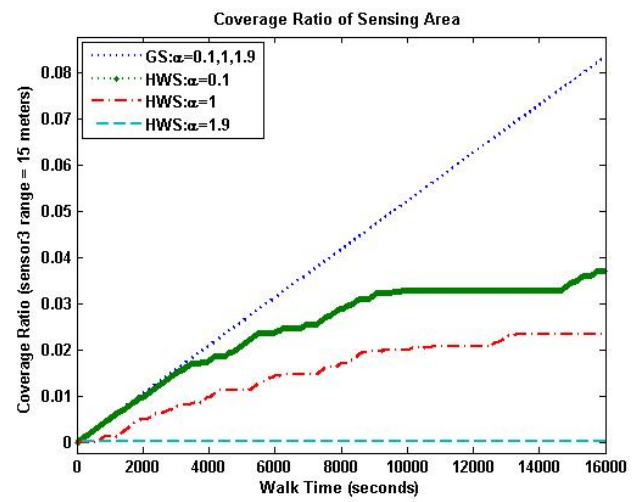

Fig. 8. Coverage Ratio-sensor3

GS technique gives better performance for $\alpha=1.9$. When human-walking velocity is neither too high nor too low, i.e when $\alpha=1$, the proposed algorithm shows better performance in terms of less battery energy consumption and spatial coverage of sensing area.

\section{CONClusion}

Human-walk velocity affects mobile phone sensing coverage and battery energy consumption. Mobile phone sensor sampling chosen according to waking speed of individual gives better performance in terms both spatial coverage and reduction of battery energy consumption. For normal walking 


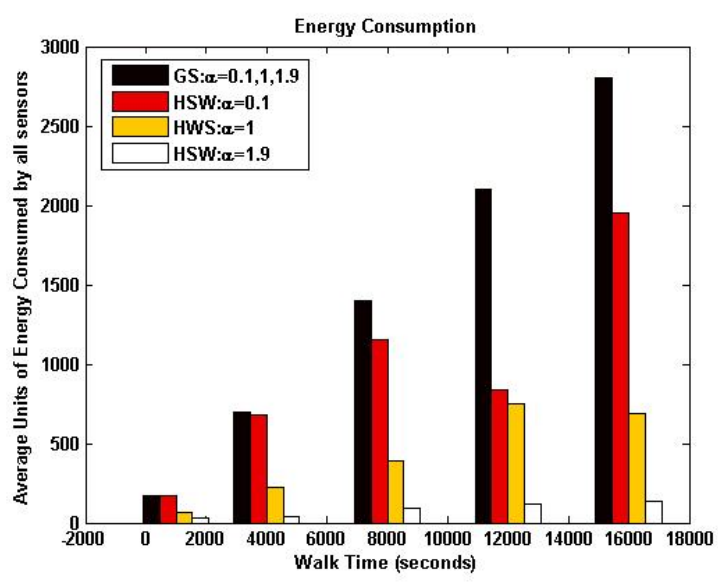

Fig. 9. Average Units of Energy Consumed by all sensors

speed of human, the proposed mobile phone sensor sampling algorithm gives better performance in terms of both battery energy reduction and area coverage. Our future work involves developing adaptive sampling and reduction of spatial overlap and battery energy consumption by considering realistic human mobility models and co-operative sensing tasks for mobile phone sensing.

\section{REFERENCES}

[1] W. Z. Khan, Y. Xiang, M. Y. Aalsalem, and Q. Arshad, "Mobile phone sensing systems: A survey," IEEE COMMUNICATIONS SURVEYS \& TUTORIALS, vol. 15, no. 1, pp. 402-427, 2013.

[2] S. S. Kanhere, "Participatory sensing: Crowdsourcing data from mobile smartphones in urban spaces," IEEE International Conference on Mobile Data Management, pp. 3-6, 2011.

[3] N. D. Lane, E. Miluzzo, H. Lu, D. Peebles, T. Choudhury, and A. T. Campbell, "A survey of mobile phone sensing," IEEE Communications Magazine, pp. 140-150, September 2010.

[4] X. Sheng, J. Tang, and W. Zhang, "Energy-efficient collaborative sensing with mobile phones," IEEE INFOCOM, pp. 1916-1924, 2012.

[5] B. WANG, "Coverage problems in sensor networks: A survey," ACM Computing Surveys, vol. 43, no. 4, pp. 32:1-32:53, October 2011.

[6] B. Liu, O. Dousse, P. Nain, and D. Towsley, "Dynamic coverage of mobile sensor networks," IEEE TRANSACTIONS ON PARALLEL AND DISTRIBUTED SYSTEMS, vol. 24, no. 2, pp. 301-311, FEBRUARY 2013.

[7] I. Rhee, M. Shin, S. Hong, K. Lee, and S. Chong, "On the levy-walk nature of human mobility," IEEE INFOCOM, pp. 1597-1605, 2008.

[8] I. Rhee, M. Shin, S. Hong, K. Lee, S. J. Kim, and S. Chong, "On the levy-walk nature of human mobility," IEEE/ACM TRANSACTIONS ON NETWORKING, vol. 19, no. 3, pp. 630-643, June 2011.

[9] B. Birand, M. Zafer, G. Zussman, and K.-W. Lee, "Dynamic graph properties of mobile networks under levy walk mobility," IEEE International Conference on Mobile Ad-Hoc and Sensor Systems, pp. 292-301, 2011.

[10] T. Karagiannis, J.-Y. L. Boudec, and M. Vojnovic, "Power law and exponential decay of inter contact times between mobile devices," MobiCom, pp. 183-194, September 2007.

[11] D. Karamshuk, C. Boldrini, M. Conti, and A. Passarella, "Human mobility models for opportunistic networks," IEEE Communications Magazine, pp. 157-165, December 2011.

[12] Networking Research Lab. Human mobility models downloads. [Online]. Available: http://research.csc.ncsu.edu/netsrv/

[13] S. Hachem, A. Pathak, and V. Issarny, "Probabilistic registration for large-scale mobile participatory sensing," IEEE International Conference on Pervasive Computing and Communications (PerCom), pp. 132 140, March 2013.

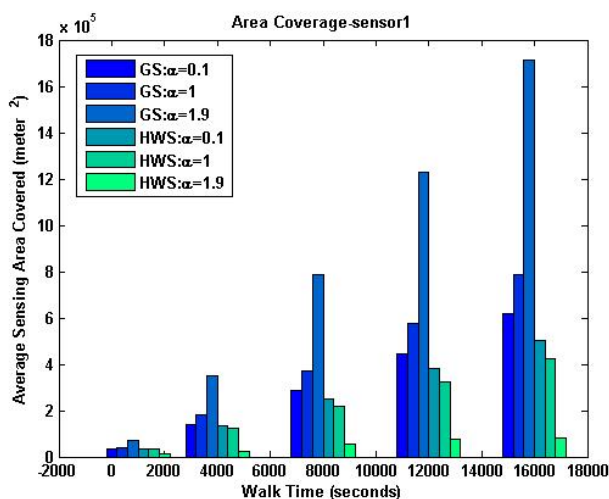

Fig. 10. Average Sensing Area Covered for Sensor 1

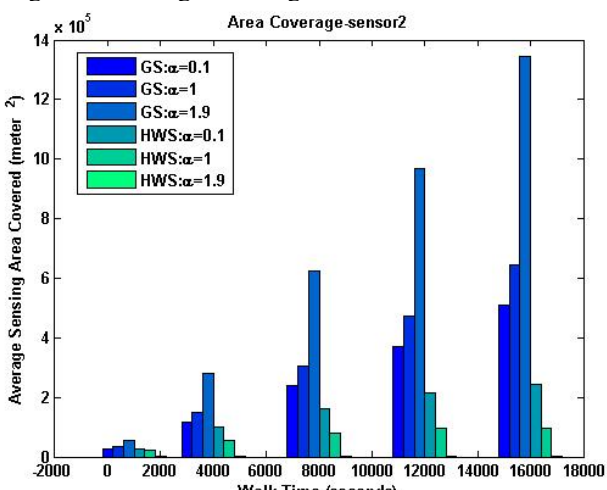

Fig. 11. Average Sensing Area Covered for Sensor2

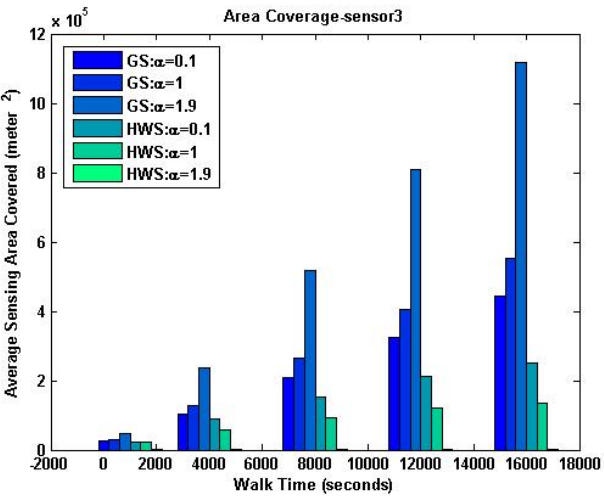

Fig. 12. Average Sensing Area Covered for Sensor3

[14] G. M. Viswanathan, S. V. Buldyrev, S. Havlin, M. G. E. da Luzk, E. P. Raposok, and H. E. Stanley, "Optimizing the success of random searches," NATURE, vol. 401, pp. 911-914, October 1999.

[15] Wikipedia. Preferred walking speed. [Online]. Available: http://en. wikipedia.org/wiki/Preferred_walking_speed

[16] T. Hara, "Quantifying sensing coverage and data delivery delay in mobile sensor networks," IEEE WCNC, pp. 481-486, 2011. 